\title{
Mortality and Multiple Causes of Death in Systemic Lupus Erythematosus - Role of the Death Certificate
}

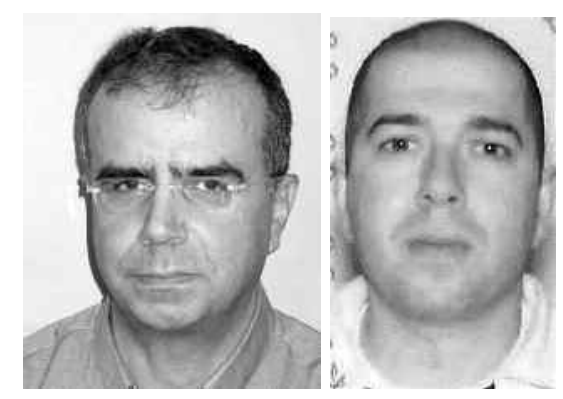

Systemic lupus erythematosus (SLE) is a chronic multisystem autoimmune disease with a wide range of laboratory and clinical features. It is characterized by a remitting recurring course. Although mild in severity in a significant number of patients, SLE can be severe and refractory to therapy in others and is associated with mortality rates higher than that of the general population.

Mortality and prognosis studies among patients with SLE have been investigated in patients referred to tertiary lupus clinics and in well defined epidemiological settings. Those studies have addressed one or more aspects of mortality and survival in SLE, including annual mortality rates, causes of death, survival rates and curves, standardized mortality ratios (SMR), and predictors associated with early and late death. The development of various valid and reproducible outcome measures in SLE has been a major step in the detecting of variables associated with morbidity and mortality in these patients. In addition, measures of SLE disease activity, permanent damage, quality of life, and other variables enable comparisons between prognostic studies from various centers ${ }^{1}$.

A review of the literature identified more than 60 large prognostic and mortality studies in SLE reported from different lupus clinics around the globe. Most studies have suggested improved survival of patients with SLE. Prognosis studies from the University of Toronto Lupus Clinic have shown that over 4 decades, the SMR has decreased from 12.6 in the first decades to 3.46 in the last decade. When SMR were stratified by entry cohort and calendar period, there was evidence of a calendar-period effect, but no cohort effect. Various disease-related variables were significantly associated with mortality but could not completely explain the trend of improved survival over calendar period observed $^{2}$.

Causes of death in SLE are classified into those related to SLE, those resulting from SLE and/or treatment-related morbidity, and those from unrelated causes. Traditionally, causes of death were categorized as primary cause of death, defined as the main disease process directly related to death, and contributing factors to mortality. This is particularly important in patients with SLE, where the distinction between the disease and its associated morbidity has not been well defined. Identifying SLE as a primary mortality cause should not be based on the disease activity index but on an SLE-related clinical manifestation that is directly related to death ${ }^{3}$.

Active SLE, infections, atherosclerotic cardiovascular disease, thrombophilia, and malignancy are the most common causes of death among patients with $\mathrm{SLE}^{3,4}$. However, a review of the reported mortality studies has identified lack of agreement with regard to the frequency of the various causes of death or the type of predictors associated with mortality. This diversity in results of mortality studies in SLE is related to differences in study design, patient populations and referral types, definitions of causes of death, and types of analyses of predictor variables for mortality.

The type of health system and insurance, early referral to tertiary centers, and geographic and economic characteristics have a significant prognostic value in SLE. However, research methodology plays a major role in identifying predictors associated with favorable or grave prognosis in $\mathrm{SLE}^{1}$.

A significant number of mortality studies have been based on retrospective analyses of charts of hospitalized patients or those seen at outpatient clinics. The patient population in this type of study comes from referral centers and not necessarily from an unselected population. Less commonly, studies are based on inception cohorts and longitudinal studies of patients with SLE followed prospectively at lupus clinics. The populations of those clinics represent a mixture of primary, secondary, and tertiary care referral. Patients are seen at fixed intervals, and their data are recorded using a standard protocol. The mortality data of those studies probably are more accurate and represent real-

See Mortality profile related to SLE, page 496 
life analyses. Such studies could be based on the data of a single center, multiple centers, or national or international registries.

In most studies, causes of death are determined by review of hospital charts, autopsy reports, and contact with the physician who cared for the patient during the terminal illness; few studies are by review of death certificates.

In this issue of The Journal, Souza, et $a l^{5}$ report a descriptive epidemiological study of causes of death among a large group of patients with a diagnosis of SLE recorded on their death certificates in the state of São Paulo, Brazil. The main characteristics of the study are population-based design, a large number of deaths, multiple causes-of-death (MCD) analyses, and analysis of trends over time.

The authors classified causes of death into underlying and contributing causes of death. This approach allows several clinical diagnoses to be included under each subcategory. This MCD analysis is unlike the classical approach, where only one primary cause of death is recorded for each death.

The data of the study indicate that patients with SLE living in the state of São Paulo die at a relatively young age, with renal disease, infections, and cardiovascular diseases being the most common causes of death. The observed number of deaths as a result of renal failure, tuberculosis, septicemia, and pneumonia were significantly higher among SLE patients compared with age- and sex-matched controls. In addition, a higher number of deaths as a result of cardiovascular diseases in general and myocardial infarction in particular occurred among patients with SLE younger than 50 years of age. Those data support what we already know: it is no longer a matter of controversy that SLE patients have an increased risk of developing cardiovascular disease, particularly before age 50 years, and accelerated atherosclerosis is a significant contributor to the morbidity and mortality of SLE.

The study data show that mean age at death has increased from 35 years during the period 1985 to 1989 to 38 years from 2003 to 2007; and the number of deaths as a result of renal failure has decreased over the years, suggesting a trend of improved survival over time.

SLE is a chronic disease characterized by a high rate of comorbid diseases, and SLE patients accumulate multiple chronic diseases during their lifespan. Not infrequently death certificates contain data on the number of comorbidities that could have contributed to death. Therefore, MCD is becoming gradually more popular, since it allows for a more precise analysis of the death cause in comparison to underlying cause of death (UCD) analysis. Utilizing the MCD approach permits assessment of the association between multiple conditions that can contribute to death. Strength of association can be measured by the ratio between the actual number of deaths for which both causes are mentioned and the number of deaths that might be expected under the assumption that the conditions were independent (the observed-to-expected ratio). In addition, the ratio of MCD to UCD can help to identify the diseases that are more important as direct contributors to the mortality ${ }^{6}$.

However, MCD methodology requires that the researcher have a clear understanding of relationships between diseases and awareness of the quality issues related to the death certificate data, i.e., potential incompleteness of data. Unlike the UCD method, where analysis is based on the single most important condition contributing to the death, which is always present on the death certificate, using the MCD method some background diseases may be omitted. Therefore, MCD analysis may underestimate the association between the secondary conditions and death.

Limitations of the Souza, et al study are related to the structure of the cohort. There is no denominator for this study. No data are given regarding the total number of patients with SLE, the disease duration, SLE-related clinical features, laboratory data, medication given to the patients, type of followup, or other patient characteristics.

This approach does not allow performance of life-table analyses, identification of predictors associated with death, or calculation of SMR. In addition, death as a result of active SLE was not defined and was not considered as a cause of death. This was missed by the authors. The role of disease activity and damage in mortality cannot be determined in this study. Identifying SLE as a primary cause of death is crucial for developing strategies for further improvement in survival of patients with SLE.

That SLE is not recorded in the death certificates of lupus patients is a major concern. In a study based on 2 lupus cohorts from North America, SLE was not recorded in $40 \%$ of death certificates, mainly among older patients and those without health insurance ${ }^{7}$. These findings highlight the limitations of vital statistics medical records in the assessment of SLE-related prognosis and epidemiological studies. During the last 2 decades paper-based health records have gradually been replaced by computer-based records. Combining the data of death certificates with those of computer-based health records will allow more accurate analyses.

In summary, despite improvement in survival rates for patients with SLE, the SMR are still 3-fold higher than for the general population. Infections, cardiovascular disease, and end-organ damage remain the major causes of death in SLE. MCD analysis has not been used in SLE. It is relatively new in prognosis studies of patients with rheumatic disease and probably will replace the classic UCD analysis. The MCD method of studying mortality in SLE will identify a better correlation between SLE activity, infection, and chronic diseases, specifically if the analyses are based on death certificates combined with data of lupus clinics or registries collected prospectively.

Personal non-commercial use only. The Journal of Rheumatology Copyright (c) 2012. All rights reserved. 
MAHMOUD ABU-SHAKRA, MD,

Rheumatic Diseases Unit,

Department of Medicine D;

VICTOR NOVACK, MD, PhD,

Clinical Research Center,

Soroka University Medical Center,

Ben-Gurion University,

Beer-Sheva, Israel

Address correspondence to Dr.Abu-Shakra; E-mail:Mahmoud@bgu.ac.il

\section{REFERENCES}

1. Abu-Shakra M. Do improved survival rates of patients with systemic lupus erythematosus reflect a global trend? J Rheumatol 2008;35:1906-8.

2. Urowitz MB, Gladman DD, Tom BD, Ibañez D, Farewell VT. Changing patterns in mortality and disease outcomes for patients with systemic lupus erythematosus. J Rheumatol 2008;35:2152-8.
3. Abu Shakra M, Urowitz MB, Gladman DD, Gough J. Mortality studies in systemic lupus erythematosus. Results from a single centre. I. Causes of death. J Rheumatol 1995;22:1259-64.

4. Bernatsky S, Boivin JF, Joseph L, Manzi S, Ginzler E, Gladman DD, et al. Mortality in systemic lupus erythematosus. Arthritis Rheum 2006;54:2550-7.

5. Souza D, Santo A, Sato E. Mortality profile related to systemic lupus erythematosus: A multiple cause-of-death analysis. J Rheumatol 2012;39:496-503.

6. Stallard E. Underlying and multiple cause mortality at advanced ages: United States 1980-1998. N Am Actuarial J 2002;6:64-87.

7. Calvo-Alén J, Alarcón GS, Campbell R Jr, Fernández M, Reveille $\mathrm{JD}$, Cooper GS. Lack of recording of systemic lupus erythematosus in the death certificates of lupus patients. Rheumatology 2005;44:1186-9.

J Rheumatol 2012;39:458-60; doi:10.3899/jrheum.111556 